\title{
Ultrashort pulse characterization with a terahertz streak camera
}

\author{
O. Schubert, ${ }^{1,2}$ C. Riek, ${ }^{1}$ F. Junginger, ${ }^{1}$ A. Sell, ${ }^{1}$ A. Leitenstorfer, ${ }^{1}$ and R. Huber ${ }^{1,2, *}$ \\ ${ }^{1}$ Department of Physics and Center for Applied Photonics, University of Konstanz, 78464 Konstanz, Germany \\ ${ }^{2}$ Department of Physics, University of Regensburg, 93040 Regensburg, Germany \\ *Corresponding author: rupert.huber@physik.uni-regensburg.de
}

Received August 26, 2011; accepted September 10, 2011;

posted October 7, 2011 (Doc. ID 153511); published November 15, 2011

A phase-locked terahertz transient is exploited as an ultrafast phase gate for femtosecond optical pulses. We directly map out the group delay dispersion of a low-power near-infrared pulse by measuring the electro-optically induced polarization rotation as a function of wavelength. Our experiment covers the spectral window from 1.0 to $1.4 \mu \mathrm{m}$ and reaches a temporal precision better than $1 \mathrm{fs}$. A quantitative analysis of the detector response confirms that this streaking technique requires no reconstruction algorithm and is also well suited for the characterization of pulses spanning more than one optical octave. (c) 2011 Optical Society of America

OCIS codes: $320.7100,190.7110,140.3295$.

The quest for ever shorter laser pulses has motivated a rapid development of sophisticated techniques for ultrafast pulse characterization [1-4]. Typically the pulse under study is superimposed with an ultrashort gate and analyzed after a nonlinear interaction with the latter. One of the pulses is delayed with respect to the other one and the experiment is repeated for different delay times.

Depending on the relative durations and carrier frequencies of the input and the gate pulses, respectively, three classes of characterization schemes may be distinguished: $(i)$ if the gate is much shorter than any feature of the pulse to be characterized, the former may directly map out the oscillating carrier field in a stroboscopic manner. This idea is routinely implemented in electrooptic detection of terahertz (THz) transients [5-7]. (ii) In the regime where both the input and the gate pulse are of similar duration and center frequency, auto- and crosscorrelation schemes have been employed $[1,3]$. These techniques are particularly popular because the gate pulse may be obtained merely by splitting the pulse under study and correlating it with its replica. High intensities required for the nonlinear interaction as well as phase distortions induced by beamsplitters, however, render this technique tedious for low-power and single-cycle near-infrared (NIR) pulses [8]. Furthermore, sophisticated projection algorithms are necessary to extract the full spectral phase. (iii) If a single cycle of the gate field is much longer than the input pulse, the former increases monotonically during the duration of the pulse under study. Exploiting material properties that depend monotonically on the applied field allows for the design of streak cameras. With electronically controlled gate fields, the maximal temporal resolution is typically bound to the picosecond regime or slower. Intense NIR laser pulses from Ti:sapphire amplifiers with stabilized carrier envelope phase, on the other hand, have accessed the attosecond time-scale [2,9]. Yet, efficient streak cameras for femtosecond pulses have not been available so far, to the best of our knowledge.

Here we present a polarization streaking technique that allows for a precise measurement of the spectral phase of low-intensity femtosecond pulses without any reconstruction algorithm. We characterize the group delay dispersion of a NIR pulse covering the wavelength range from 1.0 to $1.4 \mu \mathrm{m}$ with an average spectral energy density of $0.4 \mathrm{pJ} / \mathrm{nm}$. Our theoretical analysis demonstrates that the phase spectrum of pulses spanning multiple optical octaves may be extracted with subfemtosecond precision.

The idea of our experiment is schematically depicted in Fig. 1(a). We focus a phase-stable $\mathrm{THz}$ transient onto an electro-optic crystal. The oscillating electric field induces a birefringence via the Pockels effect which rotates the polarization of a co-propagating optical pulse in proportion to the instantaneous $\mathrm{THz}$ electric field. For a sufficiently fast variation of the gate, different temporal slices of the optical pulse experience a markedly different rotation of their polarization. Analyzing this effect as a function of wavelength is expected to yield the chirp of the input pulse directly.

To prove this principle, we generate a NIR test pulse and a THz streaking field with a multibranch Er:fiberTi:sapphire hybrid laser [10]. One branch of the femtosecond fiber laser is fed into a highly nonlinear bulk fiber to obtain an ultrabroadband continuum, with an energy of $0.2 \mathrm{~nJ}$ and a center wavelength of $1.2 \mu \mathrm{m}$ [11]. The spectral phase is controlled with an SF10 prism compressor. The pulses from another branch of the fiber system are frequency doubled and amplified in a high-power Ti:sapphire system to drive two parallel parametric amplifiers (OPAs). Difference frequency mixing of the synchronized signal trains from both OPAs has been demonstrated to result in phase-locked multi-THz transients with peak fields above $100 \mathrm{MV} / \mathrm{cm}$ [10]. In the present work, transients featuring peak fields of $1 \mathrm{MV} / \mathrm{cm}$ are sufficient, even though we record only one spectrally dispersed pulse per data point at a moderate repetition rate of $1 \mathrm{kHz}$. THz and NIR pulse trains are collinearly focused into a GaSe electro-optic sensor of a thickness of $140 \mu \mathrm{m}$ [Fig. 1(b)]. The wavelength-dependent polarization of the NIR pulse is analyzed with a Glan-Taylor prism, a monochromator, and an InGaAs photodiode. We control the relative time between input and gate pulses with a mechanical delay stage (time step: $1 \mathrm{fs}$ ) and scan the spectrum via a rotating grating. The transmission 


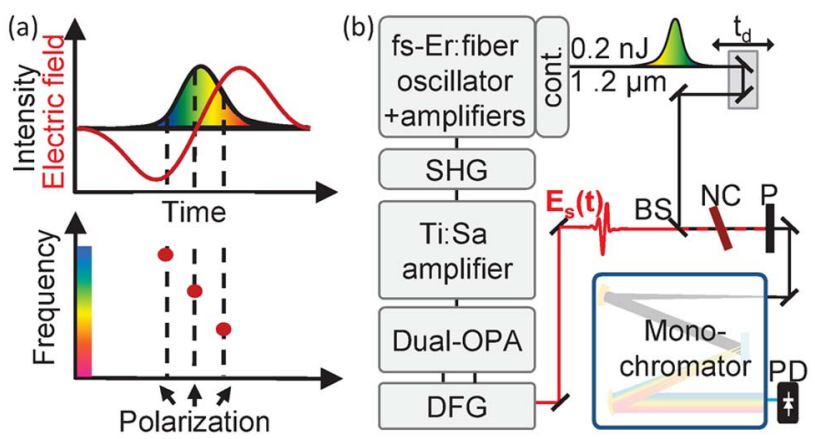

Fig. 1. (Color online) (a) Principle of THz streaking: The temporal profile of a $\mathrm{THz}$ streak field is imprinted on the polarization of a laser pulse. Polarization analysis as a function of wavelength provides the spectral group delay of the pulse. (b) Experimental setup: laser system generating NIR femtosecond pulses (cont. $=$ continuum) and $\mathrm{THz}$ transients, linear delay stage, beam splitter (BS), nonlinear crystal (NC) with applied streak field $E_{S}(t)$, polarizer $(P)$, monochromator, and photodiode (PD).

through the polarizer is compared for the situation with and without a $\mathrm{THz}$ field impinging on the electro-optic crystal. The difference $\Delta T$ is recorded by a boxcar integrator.

The intensity spectrum of the NIR pulse covers the wavelength range from below $1 \mu \mathrm{m}$ to above $1.4 \mu \mathrm{m}$ [Fig. 2(a)]. Figure 2(b) illustrates the field-induced transmission changes for two NIR-wavelengths, $1140 \mathrm{~nm}$ and $1180 \mathrm{~nm}$, as a function of the delay time. Both signals oscillate with the electric field of the applied few-cycle $\mathrm{THz}$ pulse (not shown) at a center frequency of $29 \mathrm{THz}$. The transients are identical within noise limits, but show a temporal offset of $\Delta t_{q}=13 \mathrm{fs}$. This delay is a manifestation of the spectral chirp of the NIR pulse. We follow the temporal position of a distinct feature of the THz waveform, such as a sharp zero-crossing, throughout the spectrum of the NIR pulse [dots in Fig. 2(c)]. This trace may be directly interpreted as the time-dependent instantaneous frequency of the pulse, i.e. its group delay dispersion. The parabolic shape at the short-wavelength end of the curve, between $1050 \mathrm{~nm}$ and $1150 \mathrm{~nm}$, indicates a predominantly cubic term in the phase spectrum. Above $\lambda=1150 \mathrm{~nm}$, the pulse is primarily linearly chirped. The overall phase profile leads to a pulse duration of $50 \mathrm{fs}$ (FWHM). The temporal resolution of this technique is limited by the precision of the optical delay between the THz pulse and the NIR pulse and by the accuracy at which the zero-crossing can be determined. The latter is set by the frequency of the THz field and the signal-tonoise ratio of the measurement. Fitting a sine function to the experimental data of Fig. 2(b) $(\lambda=1140 \mathrm{~nm})$ allows us to locate the zero-crossing of the transient at $t_{d}=$ $-11 \mathrm{fs}$ with an uncertainty of less than $1 \mathrm{fs}$.

While the time-domain picture offers an intuitive concept, it neglects phase-matching effects and presumes an instantaneous $\chi^{(2)}$-nonlinearity. For a more rigorous description we now switch to the frequency domain. The rotation of the polarization may be described as a sum-frequency process with type-I phase-matching. A NIR photon mixes with a THz photon to generate another NIR component polarized perpendicularly at a frequency $\omega_{\mathrm{NIR} 2}(o)=\omega_{\mathrm{NIR} 1}(e) \pm \omega_{\mathrm{THz}}(o)$. Here the indices ' $o$ ' and ' $e$ ' designate ordinary and extraordinary polarization, respectively. Since the THz frequency $\omega_{S}$ is significantly smaller than the bandwidth of the NIR pulse, the newly generated frequency overlaps coherently with the linearly transmitted input spectrum and induces an effective polarization rotation [7].

For a quantitative model we allow for an arbitrary phase of the NIR pulse and take into account sum and difference frequency processes, a frequency dependent nonlinearity [12], and phase-matching effects. We find that, for optical frequencies $\omega$ much larger than all relevant $\mathrm{THz}$ frequencies $\omega_{\mathrm{THz}}$, the group delay of the pulse may be written as [13]

$$
\frac{d \varphi_{\mathrm{NIR}}}{d \omega}=\tau_{\exp }-\tau_{\mathrm{PM}}-\tau_{\chi^{(2)}},
$$

where $\tau_{\exp }(\omega)$ represents the measured group delay. $\tau_{\mathrm{PM}}(\omega)$ and $\tau_{\chi^{(2)}}\left(\omega, \omega_{\mathrm{THz}}\right)$ account for distortions due to phase-matching effects and the frequency-dependence of the $\chi^{(2)}$-nonlinearity, respectively. The dispersion of $\chi^{(2)}$ may be neglected far off electronic or ionic resonances [14]. Thus solely phase-matching effects remain as potentially relevant factors to be corrected for. We calculate $\tau_{\mathrm{PM}}(\omega)$ from the phase-matching function [12] using the well-known dispersion of GaSe $[\underline{15}, 16]$ and subtract it from $\tau_{\exp }(\omega)$. The result, indicated by the black line in Fig. 2(b), shows virtually the same time dependence as the raw data. Thus the intuitive strategy of tracing a local feature of the $\mathrm{THz}$ transient represents an excellent approximation to the exact recovery of the full spectral phase of the NIR pulse.
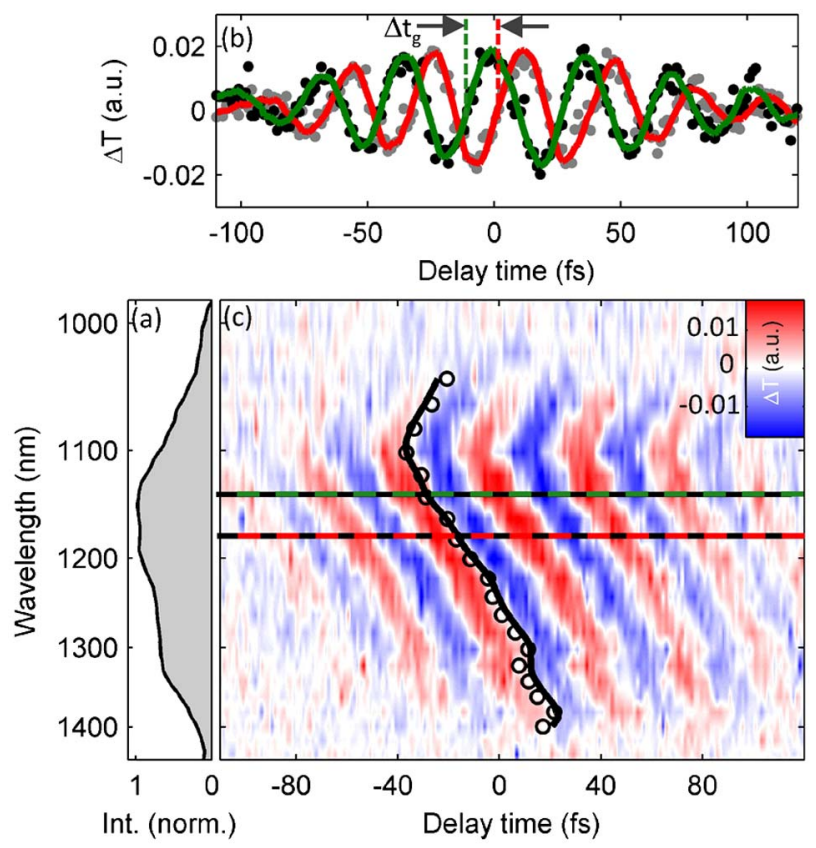

Fig. 2. (Color online) (a) Intensity spectrum of the NIR test pulse. (b) Field-induced transmission through the polarizer at $1140 \mathrm{~nm}$ (black dots) and $1180 \mathrm{~nm}$ (gray dots). (c) THz streak image. Circles indicate the temporal position of a zero-crossing of the THz waveform as a function of the NIR wavelength. The profile reflects the instantaneous frequency of the input pulse. Black line: instantaneous frequency after correction for phase mismatch. Dashed lines: cross sections presented in (b). 


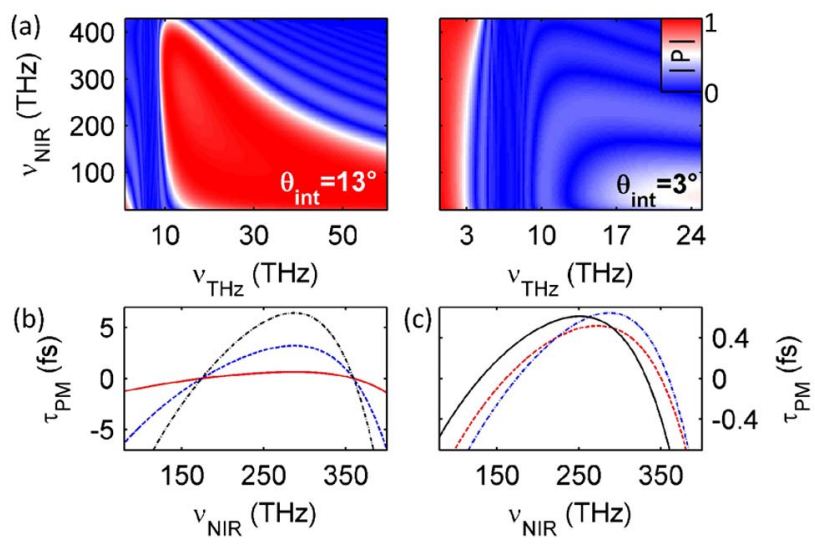

Fig. 3. (Color online) (a) Normalized amplitude of the phasematching function $P=\left|\left(e^{i \Delta \mathrm{KL}}-1\right) /(i \Delta \mathrm{KL})\right|$ for a GaSe sensor (thickness: $100 \mu \mathrm{m}$ ) at a phase-matching angle of $\theta_{\text {int }}=13^{\circ}$ (left) and $\theta_{\text {int }}=3^{\circ}$ (right). (b) Temporal offset introduced by phase mismatch for a GaSe crystal of a length of $100 \mu \mathrm{m}$ (black dashed), $50 \mu \mathrm{m}$ (blue dash-dotted), and $10 \mu \mathrm{m}$ (red solid line). (c) Temporal offset introduced for a $10 \mu \mathrm{m}$-thin GaSe crystal and gate frequencies of $1.5 \mathrm{THz}$ (red dashed line), $12 \mathrm{THz}$ (blue dash-dotted line), and $30 \mathrm{THz}$ (black solid line). The internal phase-matching angles for these frequencies are chosen as $4^{\circ}, 12^{\circ}$, and $18^{\circ}$, respectively.

Recent experimental results [8] have confirmed an urgent need for straightforward characterization schemes for pulses spanning more than one optical octave, particularly in the NIR and visible spectral domain. THz streaking may be extended to cover this regime. To illustrate this fact, we present phase-matching diagrams for a GaSe crystal (thickness: $100 \mu \mathrm{m}$ ) and two different phasematching angles [Fig. 3(a)]. For an internal propagation angle of $13^{\circ}$ with respect to the optical axis and $\omega_{\mathrm{THz}}=$ $12 \mathrm{THz}$, a phase-matching bandwidth of more than $350 \mathrm{THz}$ (FWHM) is obtained, ranging from 75 up to $425 \mathrm{THz}$. If $\omega_{\mathrm{THz}}$ is chosen below the phonon resonance, even more broadband phase-matching is possible. Typical group delay distortions $\tau_{\mathrm{PM}}$ introduced by the remaining phase mismatch are shown in Fig. 3(b) for crystal thicknesses of $100 \mu \mathrm{m}, 50 \mu \mathrm{m}$, and $10 \mu \mathrm{m}$. Even for relatively long crystals the detector response causes delays of the order of few femtoseconds, only, over a bandwidth of more than one optical octave. For a crystal length of $10 \mu \mathrm{m}$, the correction factor is within $1 \mathrm{fs}$ for a bandwidth spanning nearly two optical octaves [Fig. 3(c)] and may, thus, be safely neglected even for extremely broadband pulses. Other crystal materials, such as GaP [17], ZnTe [18,19], CdTe [20], or $\mathrm{LiNbO}_{3}$ [18], may be employed to further increase the accessible spectral range.

In conclusion we have demonstrated a versatile method for the characterization of low-intensity ultrashort laser pulses supporting bandwidths of multiple optical octaves even for relatively long nonlinear crystals. A single-shot implementation of this technique is feasible in a way similar to single-shot detection of $\mathrm{THz}$ transients [21-24] by replacing the photodiode with a photodiode array and recording spectra for one delay time only. No reconstruction algorithm is required to extract the spectral phase of the pulses. Finally we stress that even though we have applied relatively high $\mathrm{THz}$ fields in our present study, the presented method will work wherever electro-optic sampling is applicable. Further experiments with yet more broadband pulses and compact laser sources at high repetition rates are currently under way.

We wish to thank D. Brida for fruitful discussions. Support by the German Research Foundation (DFG) via the Emmy Noether Program is gratefully acknowledged.

\section{References and Notes}

1. R. Trebino, P. Bowlan, P. Gabolde, X. Gu, S. Akturk, and M. Kimmel, Laser Photon. Rev. 3, 314 (2009).

2. J. Itatani, F. Quere, G. L. Yudin, M. Y. Ivanov, F. Krausz, and P. B. Corkum, Phys. Rev. Lett. 88, 173903 (2002).

3. I. Z. Kozma, P. Baum, U. Schmidhammer, S. Lochbrunner, and E. Riedle, Rev. Sci. Instrum. 75, 2323 (2004).

4. F. Cilento, C. Giannetti, G. Ferrini, S. Dal Conte, T. Sala, G. Coslovich, M. Rini, A. Cavalleri, and F. Parmigiani, Appl. Phys. Lett. 96, 021102 (2010).

5. R. Huber, A. Brodschelm, F. Tauser, and A. Leitenstorfer, Appl. Phys. Lett. 76, 3191 (2000).

6. K. Liu, J. Xu, and X.-C. Zhang, Appl. Phys. Lett. 85, 863 (2004)

7. C. Kübler, R. Huber, S. Tübel, and A. Leitenstorfer, Appl. Phys. Lett. 85, 3360 (2004).

8. G. Krauss, S. Lohss, T. Hanke, A. Sell, S. Eggert, R. Huber, and A. Leitenstorfer, Nat. Photon. 4, 33 (2010).

9. Y. Mairesse and F. Quere, Phys. Rev. A 71, 011401 (2005).

10. A. Sell, A. Leitenstorfer, and R. Huber, Opt. Lett. 33, 2767 (2008).

11. A. Sell, G. Krauss, R. Scheu, R. Huber, and A. Leitenstorfer, Opt. Express 17, 1070 (2009).

12. R. W. Boyd, Nonlinear Optics (Academic, 2008).

13. A more detailed derivation of the detector response will be presented elsewhere.

14. Note that the dependence of $\chi^{(2)}$ on $\omega_{S}$ only leads to a constant time delay independent of $\omega$.

15. C.-W. Chen, T.-T. Tang, S.-H. Lin, J. Y. Huang, C.-S. Chang, P.-K. Chung, S.-T. Yen, and C.-L. Pan, J. Opt. Soc. Am. B 26, A58 (2009).

16. K. L. Vodopyanov and L. A. Kulevskiib, Opt. Commun. 118, 375 (1995).

17. Q. Wu and X.-C. Zhang, Appl. Phys. Lett. 70, 1784 (1997)

18. C. Winnewisser, P. Uhd Jepsen, M. Schall, V. Schyja, and H. Helm, Appl. Phys. Lett. 70, 3069 (1997).

19. A. Nahata, J. T. Yardley, and T. F. Heinz, Appl. Phys. Lett. 75, 2524 (1999).

20. F. Z. Meng, M. D. Thomson, D. Molter, T. Löffler, J. Jonuscheit, R. Beigang, J. Bartschke, T. Bauer, M. Nittmann, and H. G. Roskos, Opt. Express 18, 11316 (2010).

21. Z. Jiang and X.-C. Zhang, Appl. Phys. Lett. 72, 1945 (1998).

22. F. G. Sun, Z. Jiang, and X.-C. Zang, Appl. Phys. Lett. 73, 2233 (1998).

23. J. Shan, A. S. Weling, E. Knoesel, L. Bartels, M. Bonn, A. Nahata, G. A. Reider, and T. F. Heinz, Opt. Lett. 25, 426 (2000).

24. H. Matlis, G. R. Plateau, J. van Tilborg, and W. P. Leemans, J. Opt. Soc. Am. B 28, 23 (2011). 